\title{
A GENERAL TRANSFER FUNCTION REPRESENTATION FOR A CLASS OF HYPERBOLIC DISTRIBUTED PARAMETER SYSTEMS
}

\author{
KRZYSZTOF BARTECKI \\ Institute of Control and Computer Engineering \\ Opole University of Technology, ul. Sosnkowskiego 31, 45-272 Opole, Poland \\ e-mail:k.bartecki@po.opole.pl
}

\begin{abstract}
Results of transfer function analysis for a class of distributed parameter systems described by dissipative hyperbolic partial differential equations defined on a one-dimensional spatial domain are presented. For the case of two boundary inputs, the closed-form expressions for the individual elements of the $2 \times 2$ transfer function matrix are derived both in the exponential and in the hyperbolic form, based on the decoupled canonical representation of the system. Some important properties of the transfer functions considered are pointed out based on the existing results of semigroup theory. The influence of the location of the boundary inputs on the transfer function representation is demonstrated. The pole-zero as well as frequency response analyses are also performed. The discussion is illustrated with a practical example of a shell and tube heat exchanger operating in parallel- and countercurrent-flow modes.
\end{abstract}

Keywords: distributed parameter system, hyperbolic system, partial differential equation, transfer function, heat exchanger.

\section{Introduction}

Distributed Parameter Systems (DPSs) represent a large class of dynamical systems in which process variables depend not only on time but also on the spatial variables, which express the geometry of the system. In practice, almost all industrial processes fall into this category, while the existence of the so-called Lumped Parameter Systems (LPSs) results mainly from the adoption of a simplified model of the reality, in which spatial effects are neglected or averaged. Typical examples of a DPS include heat transfer and fluid flow phenomena, as well as processes occurring in chemical reactors, semiconductor manufacturing, polymer processing, air pollution monitoring and many others (Friedly, 1972; Wu and Liou, 2001; Bounit, 2003; Bartecki, 2007; 2009; Zavala-Rio et al., 2009; Li and Qi 2010; Ucinski, 2012; Patan, 2012).

Based on the phenomenological models of the processes, established usually on the basis of the mass or energy conservation balance laws, one obtains their mathematical description, mostly in the form of Partial Differential Equations (PDEs) (Evans, 1998; Mattheij et al., 2005). Depending on the nature of the modeled phenomena, the equations can be of the parabolic type (which are typical for unsteady heat conduction and diffusion problems), hyperbolic type (representing convection, advection and wave propagation phenomena) or elliptic type (describing steady-state physical phenomena, e.g., electrostatic, magnetostatic or gravitational fields). Mathematical models of DPSs obtained on the basis of PDEs are described in an infinite-dimensional state space, usually in a Hilbert space, and their transfer functions have the form of irrational functions, as opposed to the rational ones describing the dynamic properties of LPSs (Callier and Winkin, 1993; Rabenstein, 1999; Grabowski and Callier, 2001a; Zwart, 2004; Curtain and Morris, 2009).

Comparative analyses of rational and irrational transfer functions show many essential differences between them, and one of the most important concerns the number of their poles and zeros. In the case of rational transfer functions, the number of poles and zeros is finite and corresponds to the number of polynomial roots appearing in the numerator and the denominator of the transfer function, respectively. On the other hand, irrational transfer functions, due to the presence of functions which are periodic for the imaginary argument (as, e.g., exponential or hyperbolic ones), may have an infinite number of poles and/or zeros. However, some of them have neither poles nor zeros, as is in the case of the well-known transfer function of a time delay system, 
$G(s)=\mathrm{e}^{-\tau s}$. Another striking difference concerns the behavior of the transfer functions in the limit cases, e.g., when the argument $s$ tends to infinity. In the case of proper rational functions the limit is zero, while for irrational ones the limits at the infinity may depend on the chosen direction on the complex plane. For example, in the case of the above-mentioned transfer function $G(s)=e^{-\tau s}$, the limit at infinity calculated along the real axis equals zero, while calculated along the imaginary one does not exist due to the periodicity of the exponential function for the imaginary argument (Curtain and Morris, 2009).

The knowledge of transfer functions enables the design of control systems using frequency response techniques, and this statement refers both to the LPS and the DPS (Jovanović and Bamieh, 2006). However, due to the mathematical complexity and the above-mentioned peculiarities of irrational transfer functions, their analysis is more difficult, and possible applications more limited than in the case of finite-dimensional models. Therefore, in order to enable the implementation of the techniques developed over the years and still commonly used for the synthesis of control systems, infinite-dimensional DPS models are usually replaced by their finite-dimensional approximations (Filbet and Shu, 2005; Contou-Carrere and Daoutidis, 2008; Ding et al., 2009; Jones and Kerrigan, 2010). These approximations often require a further reduction using, for example, techniques based on the Principal Component Analysis (PCA) (Park and Cho, 1996; Li and Qi, 2010; Bartecki, 2011; 2012a; 2012b). However, regardless of the approximation method used, the starting point for the synthesis of a control system should be based on a possibly accurate description of the DPS, taking into account its infinite-dimensional nature, e.g., a model in the form of an irrational transfer function.

The paper presents the results of transfer function analysis for a certain class of DPSs in which the mass, heat and energy transport phenomena occur. This class of systems, among which one can mention, e.g., heat exchangers, transport pipelines, irrigation channels or electrical transmission lines, is usually described by a PDE of the hyperbolic type and known under the common name of the hyperbolic systems of conservation laws (Friedly, 1972; Delnero et al., 2004; Bounit, 2003; Kowalewski, 2009; Bartecki and Rojek, 2005; Bartecki, 2007; 2009). Their dynamical properties have been intensively studied in the literature in recent years. For example, Zwart et al. (2010) prove that hyperbolic boundary control systems are well-posed if and only if the state operator generates a strongly continuous one-parameter semigroup. Furthermore, they show that the corresponding transfer function is regular, i.e., has a limit for the argument $s$ going to infinity. Litrico and Fromion (2009a; 2009b) use a frequency domain method for the boundary control of hyperbolic conservation laws and show that the resulting transfer functions belong to the so-called Callier-Desoer algebra, for which the Nyquist theorem provides necessary and sufficient conditions for the input-output closed-loop stability. The problems of robust control of hyperbolic PDE systems have been studied extensively, e.g., by Christofides and Daoutidis (1998a; 1998b) or Litrico et al. (2007). Interesting results on the stability and well-posedness analysis of the class of systems considered were presented recently, e.g., by $\mathrm{Xu}$ and Sallet (2002), Bounit (2003), Coron et al. (2007), Dos Santos et al. (2008), Chentouf and Wang (2009), Diagne et al. (2012), as well as Jacob and Zwart (2012).

The structure of the article is as follows. After Introduction, Section 2 reviews the mathematical model of the discussed class of DPSs in the form of a set of PDEs and formulates its hyperbolicity conditions. Next, the analysis is focused on the systems with two distributed state variables and boundary-type control. Based on the semigroup approach, some known theoretical results of well-posedness and stability for the given class of dissipative hyperbolic systems are recalled. Section 3 starts with a discussion of some known properties of the transfer functions of DPSs with boundary inputs. The closed-form expressions for the individual elements of the $2 \times 2$ transfer function matrix are derived based on the decoupled canonical system of two hyperbolic PDEs. In Section 4, a shell and tube heat exchanger operating in parallel- and countercurrent-flow modes is presented as a typical example of the hyperbolic DPS with boundary inputs considered. Based on the derived transfer functions, selected frequency responses of the heat exchanger are also presented here. The article concludes with a summary of new results and directions for further research.

\section{Hyperbolic systems}

2.1. General case. Many of the above-mentioned DPSs can be described, after possible linearization in a given operating point, by the following system of linear homogeneous PDEs of the first order (see Christofides and Daoutidis, 1998b; Evans, 1998; Strikwerda, 2004; Mattheij et al., 2005; Chentouf and Wang, 2009; Diagne et al., 2012):

$$
E \frac{\partial w(l, t)}{\partial t}+F \frac{\partial w(l, t)}{\partial l}=W w(l, t),
$$

where $w(l, t): Q \rightarrow \mathbb{R}^{n}$ is a vector function representing the spatio-temporal distribution of the $n$ state variables

$$
w(l, t)=\left[\begin{array}{llll}
w_{1}(l, t) & w_{2}(l, t) & \ldots & w_{n}(l, t)
\end{array}\right]^{T},
$$

defined on a set $Q=\Omega \times \Theta$, where $\Omega=[0, L] \subset \mathbb{R}$ is the domain of the spatial variable $l, \Theta=[0,+\infty) \subset \mathbb{R}$ is the domain of the time variable $t$, and $E, F, W \in \mathbb{R}^{n \times n}$ are matrices with constant entries. 
Assuming that for $\operatorname{det}(E) \neq 0$ and $\operatorname{det}(F) \neq 0$ there exists a non-singular transformation matrix $S \in \mathbb{R}^{n \times n}$ such that the following equation holds:

$$
S^{-1} F E^{-1} S=\Lambda,
$$

where $\Lambda$ is a diagonal matrix, pre-multiplying both the sides of (1) by $S^{-1}$ and using the following identity

$$
E^{-1} S S^{-1} E \equiv I,
$$

Eqn. (1) can be transformed into the form

$$
\begin{gathered}
S^{-1} E \frac{\partial w(l, t)}{\partial t}+S^{-1} F E^{-1} S S^{-1} E \frac{\partial w(l, t)}{\partial l} \\
=S^{-1} W w(l, t) .
\end{gathered}
$$

Then, taking into account (3) and (4) and introducing the transformed state variable vector $x(l, t)$ defined as

$$
x(l, t)=S^{-1} E w(l, t),
$$

Eqn. (1) can be finally written as follows:

$$
\frac{\partial x(l, t)}{\partial t}+\Lambda \frac{\partial x(l, t)}{\partial l}=K x(l, t),
$$

where

$$
K=S^{-1} W E^{-1} S
$$

Remark 1. Owing to the diagonal form of the matrix $\Lambda$, each equation of the system (7) contains both temporal and spatial derivatives of the same state variable $x_{i}(l, t)$, for $i=1,2, \ldots, n$. Therefore, this system is commonly referred to as decoupled or weakly coupled, i.e., coupled only through the terms that do not contain derivatives, as opposed to the original system (1) which in general can be strongly coupled.

Definition 1. (Mattheij et al., 2005) The system (1) is said to be hyperbolic if all the eigenvalues of the matrix $F E^{-1}$ are real and different from zero. Additionally, if all the eigenvalues are distinct, then Eqn. (1) is said to be strictly hyperbolic.

Therefore, the strict hyperbolicity of the system means that the matrix $\Lambda$ in (7) takes the following form:

$$
\Lambda=\operatorname{diag}\left(\lambda_{1}, \ldots, \lambda_{p}, \lambda_{p+1}, \ldots, \lambda_{n}\right),
$$

where $\lambda_{i} \in \mathbb{R} \backslash\{0\}$ for $i=1,2, \ldots, n$, represents the eigenvalues of the matrix $F E^{-1}$ arranged in descending order,

$$
\lambda_{1}>\ldots>\lambda_{p}>0>\lambda_{p+1}>\ldots>\lambda_{n},
$$

with $p \leq n$ representing the number of positive eigenvalues.

The matrix $S$ in (3) can be presented as follows:

$$
S=\left[\begin{array}{llllll}
s_{1} & \ldots & s_{p} & s_{p+1} & \ldots & s_{n}
\end{array}\right],
$$

where $s_{i} \in \mathbb{R}^{n}$ for $i=1,2, \ldots, n$ denotes the corresponding column eigenvectors of the matrix $F E^{-1}$.
Remark 2. If the matrix $F E^{-1}$ is symmetric, then the system (1) is hyperbolic. In the case of hyperbolic PDEs describing physical phenomena mentioned in Section 1 its eigenvalues usually represent the wave propagation velocities (as for the electrical transmission line) or mass and energy transport rates (as for the shell and tube heat exchanger).

2.2. Initial and boundary conditions. In order to obtain a unique solution of (7), one must specify the appropriate initial and boundary conditions. The initial conditions represent the initial (i.e., determined for $t=0$ ) distribution of the values of all $n$ state variables for the whole set $\Omega$,

$$
x(l, 0)=x_{0}(l),
$$

where $x_{0}(l): \Omega \rightarrow \mathbb{R}^{n}$ is a given vector function.

On the other hand, the boundary conditions represent the requirements to be met by the solution $x(l, t)$ at the boundary points of $\Omega$. In general, these conditions may take the form of a linear combination of the Dirichlet and Neumann boundary conditions, as the so-called boundary conditions of the third kind (Dooge and Napiorkowski, 1987; Christofides and Daoutidis, 1997; Sutherland and Kennedy, 2003; Ancona and Coclite, 2005). For the class of hyperbolic systems considered, we assume the Dirichlet boundary conditions, which can be written in the following compact way (see $\mathrm{Xu}$ and Sallet, 2002; Diagne et al., 2012):

$$
\left[\begin{array}{l}
x^{+}(0, t) \\
x^{-}(L, t)
\end{array}\right]=\left[\begin{array}{ll}
P_{00} & P_{01} \\
P_{10} & P_{11}
\end{array}\right]\left[\begin{array}{l}
x^{+}(L, t) \\
x^{-}(0, t)
\end{array}\right]+\left[\begin{array}{l}
R_{0} \\
R_{1}
\end{array}\right] u(t)
$$

with

$$
x^{+}=\left[\begin{array}{c}
x_{1} \\
\vdots \\
x_{p}
\end{array}\right], \quad x^{-}=\left[\begin{array}{c}
x_{p+1} \\
\vdots \\
x_{n}
\end{array}\right] .
$$

The vector function $u(t) \in L^{2}\left([0,+\infty) ; \mathbb{R}^{r}\right)$ in 13 expresses the inhomogeneity of the boundary conditions which can be identified with $r$ external inputs to the system, including control signals as well as external disturbances. The constant matrices $P_{00} \in \mathbb{R}^{p \times p}, P_{01} \in$ $\mathbb{R}^{p \times(n-p)}, P_{10} \in \mathbb{R}^{(n-p) \times n}, P_{11} \in \mathbb{R}^{(n-p) \times(n-p)}$ express boundary feedbacks and reflections, whereas $R_{0} \in \mathbb{R}^{p \times r}$ and $R_{1} \in \mathbb{R}^{(n-p) \times r}$ represent the effect of the external inputs $u(t)$ on the boundary conditions $x^{+}(0, t)$ and $x^{-}(L, t)$, respectively.

2.3. Second-order systems. Among many different kinds of DPSs, an important class is constituted by the systems with two spatio-temporal state variables which can be described, after appropriate assumptions, by a second-order hyperbolic PDE. The following typical 
examples can be mentioned here (Górecki et al., 1989; Gvozdenac, 1990; Miano and Maffucci, 2001; Arbaoui et al., 2007; Dos Santos et al., 2008; Bartecki, 2009):

- the voltage $u(l, t)$ and the current $i(l, t)$ in the electrical transmission line,

- the pressure $p(l, t)$ and the flow $q(l, t)$ of the medium transported through the pipeline,

- the temperatures $\vartheta_{1}(l, t)$ and $\vartheta_{2}(l, t)$ of the heating and the heated fluid in the case of a shell and tube heat exchanger.

In the case of the above-mentioned systems, Eqn. (7) takes, after possible previous linearization and diagonalization, the form of the following two PDEs:

$$
\begin{aligned}
& \frac{\partial x_{1}(l, t)}{\partial t}+\lambda_{1} \frac{\partial x_{1}(l, t)}{\partial l}=k_{11} x_{1}(l, t)+k_{12} x_{2}(l, t), \\
& \frac{\partial x_{2}(l, t)}{\partial t}+\lambda_{2} \frac{\partial x_{2}(l, t)}{\partial l}=k_{21} x_{1}(l, t)+k_{22} x_{2}(l, t),
\end{aligned}
$$

where $k_{11}, k_{12}, k_{21}, k_{22}$ are constant elements of the matrix $K$ given by (8).

It is assumed here that the only external influence on the state variables is given by the boundary conditions (13) and no boundary feedbacks nor reflections are present in the system, i.e., $P_{00}, P_{01}, P_{10}, P_{11}$ in (13) are all zero matrices. The second important assumption is that the system is given directly by the two weekly coupled PDEs (15) and 16, or is considered in terms of the transformed state variables (6). Two cases often occurring in practice are studied here: in the first one, both boundary conditions are given for the same edge $(l=0)$ of $\Omega$, and in the second the input function $u(t)$ acts on the two different edges, $l=0$ and $l=L$, respectively. Such a situation occurs, e.g., in the case of shell and tube heat exchangers, for which two different flow configurations are possible (Bartecki and Rojek, 2005; Bartecki, 2007; Delnero et al., 2004; Gvozdenac, 1990; Zavala-Río et al., 2009; Maidi et al., 2010). Therefore, two new definitions are now introduced in order to discriminate between the two above-mentioned classes of boundary inputs.

Definition 2. The boundary inputs of the system (15) and (16) will be referred to as congruent for the following parameter values of Eqn. (13): $n=l=p=2$ and $R_{0}=$ $I_{2}$, which leads to the input vector of the form

$$
u(t)=\left[\begin{array}{ll}
u_{1}(t) & u_{2}(t)
\end{array}\right]^{T}=\left[x_{1}(0, t) \quad x_{2}(0, t)\right]^{T} .
$$

Definition 3. The boundary inputs to the system (15) and (16) will be referred to as incongruent for the following parameter values of Eqn. (13): $n=l=2, p=1, R_{0}=$
[1 0 ] and $R_{1}=\left[\begin{array}{ll}0 & 1\end{array}\right]$, which leads to the input vector of the form

$$
u(t)=\left[\begin{array}{ll}
u_{1}(t) & u_{2}(t)
\end{array}\right]^{T}=\left[x_{1}(0, t) \quad x_{2}(L, t)\right]^{T} .
$$

Remark 3. Taking into account (10), it can be noticed that the congruent boundary inputs should be imposed for $\lambda_{1}>0$ and $\lambda_{2}>0$, while the incongruent ones for $\lambda_{1}>0$ and $\lambda_{2}<0$.

The above assumptions about the form of the boundary conditions representing the external influences on the system have their practical reasons. For example, in the case of the above-mentioned shell and tube heat exchanger operating in the so-called parallel-flow mode, the temperatures of the heated and the heating fluid are given for the same geometric point of the exchanger. On the other hand, the temperatures of the fluids flowing into the exchanger operating in the countercurrent-flow mode are specified for its two opposite sides.

2.4. State and output equations. Ignoring for the moment the boundary inputs, Eqn. (1) can be written in the following homogeneous abstract Cauchy form (Curtain and Zwart, 1995):

$$
\frac{\mathrm{d} x(t)}{\mathrm{d} t}=A x(t), \quad x(0)=x_{0},
$$

with the state variable $x(t)$ belonging to the Hilbert space $X=L^{2}\left(\Omega, \mathbb{R}^{n}\right)$ and the operator $A: X \supset D(A) \rightarrow X$ which can be expressed based on (7) as

$$
A h=-\Lambda \frac{\mathrm{d} h}{\mathrm{~d} l}+K h .
$$

Considering the second-order system (15) and (16), the domain $D(A)$ is given for $h=\left[h_{1}, h_{2}\right]^{T}$ by

$$
D(A)=\left\{h \in H^{1}(\Omega) \times H^{1}(\Omega) \mid h_{1}(0)=h_{2}(0)=0\right\},
$$

for $\lambda_{1}>0, \lambda_{2}>0$, and by

$$
D(A)=\left\{h \in H^{1}(\Omega) \times H^{1}(\Omega) \mid h_{1}(0)=h_{2}(L)=0\right\},
$$

for $\lambda_{1}>0, \lambda_{2}<0$, with $H^{1}(\Omega)$ the Hilbert-Sobolev space of functions, whose distributional derivatives lie in $L^{2}(\Omega)$.

Assumption 1. The matrix $K$ in (7) is symmetric and negative-semidefinite, i.e., it fulfills $\xi^{T} K \xi \leq 0$ for any $\xi \in \mathbb{R}^{n}$.

Remark 4. Assumption 1, together with the proper boundary conditions, ensures the so-called dissipativity of the operator $A$ in 20) (Phillips, 1957; Lumer and Phillips, 1961; Xu and Sallet, 2002; Chentouf and Wang, 2009; Tucsnak and Weiss, 2006). This means that the associated physical model has no internal energy sources 
and the boundary conditions are such that no energy enters the model through the boundaries. These conditions are satisfied by many chemical engineering systems like tubular reactors and heat exchangers.

Theorem 1. (Rauch and Taylor, 1974; Lumer and Phillips, 1961) Let the assumptions on the hyperbolicity be satisfied for the system (7). Then for each $x_{0} \in X$ the system has a unique solution

$$
x(\cdot, t) \in C([0,+\infty) ; X) .
$$

Moreover, if the system is dissipative, the semigroup of bounded linear operators $T(t)$ from $X$ into itself such that $x(\cdot, t)=T(t) x_{0}$, generated by the operator $A(20)$, is contractive, i.e., it fulfills

$$
\|T(t)\|_{\mathcal{L}(X)} \leq 1 \text { for all } t \geq 0 .
$$

Considering the second-order system (15) and (16), the boundary inputs introduced in Definitions 2 and 3 can be included as an inhomogeneity into (19), giving as a result the following state equation (see Curtain and Weiss, 2006):

$$
\frac{\mathrm{d} x(t)}{\mathrm{d} t}=A x(t)+B u(t), \quad x(0)=x_{0},
$$

where $u(t) \in L^{2}\left([0,+\infty) ; \mathbb{R}^{2}\right)$, and

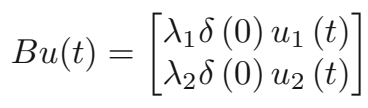

or

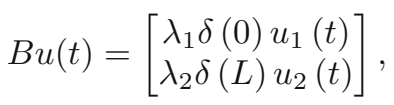

where $B$ is the control operator for the case of the congruent and incongruent boundary inputs, respectively. In the above formulation, the input points are modeled as a Delta distribution at the extremities $l=0$ and $l=L$ (see Tucsnak and Weiss, 2006; 2009; Maidi et al., 2010).

The state equation (25) can be completed by the following output equation:

$$
y(t)=C x(t),
$$

where $y$ is in the Hilbert space $Y$ and $C$ is a linear output (observation) operator. This operator can be taken as identity, giving as a result a "distributed output", i.e.,

$$
y(t)=x(t)
$$

or as a pointwise output operator with

$$
C x(t)=\left[\begin{array}{l}
\int_{0}^{L} \delta(L) x_{1}(\xi, t) \mathrm{d} \xi \\
\int_{0}^{L} \delta(L) x_{2}(\xi, t) \mathrm{d} \xi
\end{array}\right]=\left[\begin{array}{l}
x_{1}(L, t) \\
x_{2}(L, t)
\end{array}\right]
$$

or

$$
C x(t)=\left[\begin{array}{l}
\int_{0}^{L} \delta(L) x_{1}(\xi, t) \mathrm{d} \xi \\
\int_{0}^{L} \delta(0) x_{2}(\xi, t) \mathrm{d} \xi
\end{array}\right]=\left[\begin{array}{c}
x_{1}(L, t) \\
x_{2}(0, t)
\end{array}\right],
$$

which can be treated as "opposite boundary" counterparts of the input operators (26) and (27), with $y(t) \in$ $L^{2}\left([0,+\infty) ; \mathbb{R}^{2}\right)$.

Although the above formulation of input and output operators seems to be soundly justified by practical considerations (e.g., inlet and outlet points of a shell and tube heat exchanger are usually located on its extreme opposite ends), it causes some mathematical difficulties since $B$ and $C$ are unbounded, $\delta(0), \delta(L) \notin L^{2}(\Omega)$. However, as will be shown in the next section, the notion of the transfer function can be preserved for some class of distributed parameter systems with a certain unboundedness of the control and observation operators.

\section{Transfer function description}

\subsection{Definition and properties.}

Definition 4. (Curtain and Zwart, 1995) The transfer function $G(s)$ of the linear system described by the state and output equations (25) and (28), is the $\mathcal{L}\left(\mathbb{R}^{2}, \mathbb{R}^{2}\right)$-valued function of a complex variable given by

$$
y(s)=G(s) u(s) \text { for } \operatorname{Re}(s)>\alpha,
$$

where $y(s)$ and $u(s)$ are the Laplace transforms of $y(t)$ and $u(t)$, respectively, and $\alpha$ is a real parameter such that $\mathrm{e}^{-\alpha} u(\cdot) \in L^{2}\left([0,+\infty) ; \mathbb{R}^{2}\right)$ produces $\mathrm{e}^{-\alpha} y(\cdot) \in$ $L^{2}\left([0,+\infty) ; \mathbb{R}^{2}\right)$ for every $u$.

Assuming temporarily that both $B$ and $C$ are bounded linear operators: $B \in \mathcal{L}\left(\mathbb{R}^{2}, X\right)$ and $C \in$ $\mathcal{L}\left(X, \mathbb{R}^{2}\right)$, the transfer function $G(s)$ of the system is given by (Callier and Winkin, 1993; Curtain and Zwart, 1995)

$$
G(s)=C(s I-A)^{-1} B \text { for } \operatorname{Re}(s)>\omega_{0},
$$

where $(s I-A)^{-1} \in \mathcal{L}(X, D(A))$ is the resolvent of $A$, i.e., the Laplace transform of the semigroup $T(t)$ generated by $A$, and $\omega_{0}$ is the growth constant of this semigroup.

The above definition of the transfer function can be extended to a more general class of systems, where some unboundedness of the input and output operators is possible, as shown by Salamon (1987), Curtain et al. (1992), Callier and Winkin (1993), Grabowski and Callier (2001a; 2001b), or Cheng and Morris (2003). Such general systems are interesting since they allow, e.g., the exact boundary control as well as the pointwise 
observation, as considered in Section 2.4. To this end, the notion of the so-called admissibility of the input and output operators has been introduced in the literature. The mild solution of (25) and (28) can be written, assuming $t \geq 0$, in the following general form (Curtain and Zwart, 1995):

$$
\begin{aligned}
& x(t)=T(t) x_{0}+\int_{0}^{t} T(t-\tau) B u(\tau) \mathrm{d} \tau, \\
& y(t)=C T(t) x_{0}+C \int_{0}^{t} T(t-\tau) B u(\tau) \mathrm{d} \tau,
\end{aligned}
$$

where $T(t)$ is the semigroup generated by $A(20)$. Without going too far into details, it may be stated that the operator $B$ for which the integral term in (34) lies in $X$ for every $u \in L^{2}\left(\left[0, t_{1}\right], \mathbb{R}^{2}\right)$ and some $t_{1}>0$ is called an admissible input operator. Similarly, the operator $C$ for which one can uniquely define a function $y \in L^{2}\left(\left[0, t_{1}\right], \mathbb{R}^{2}\right)$ for some $t_{1}>0$ and all $x_{0} \in X$, is called an admissible output operator (Curtain et al., 1992; Weiss, 1994; Staffans and Weiss, 2000).

If both $B$ and $C$ are admissible operators, the system (34) and (35) is called a Pritchard-Salamon system (Pritchard and Salamon, 1984; Curtain et al., 1992). Since the admissibility of the studied unbounded operators $B$ and $C$ for the semigroup $T(t)$ generated by $A$ in (20) has been already proved (see, e.g., Tucsnak and Weiss, 2006) one may state that the dissipative hyperbolic systems considered belong to the Pritchard-Salamon class. Moreover, as proved by Curtain (1988), if $A$ generates a $C_{0}$-semigroup $T(t)$, either $\mu$-exponential stabilizability or $\mu$-exponential detectability of this semigroup is sufficient for the given systems to belong to the Callier-Desoer class $\mathcal{B}(\mu)$, for which the Nyquist theorem provides necessary and sufficient conditions for input-output closed-loop stability (Callier and Winkin, 1993; Curtain and Zwart, 1995; Sasane, 2002). The above statement holds true even if $B$ and $C$ are unbounded operators, which is the case considered here. The same has been proved by Litrico and Fromion (2009a) for a very particular hyperbolic system of conservation laws, based on the transfer function decomposition into an unstable finite dimensional part and a stable infinite dimensional part.

From the semigroup analysis performed in Section 2.4 we have that the transfer functions of the hyperbolic systems considered are in $H_{\infty}$, i.e., they are analytic and bounded in the open right half of the complex plane, $\operatorname{Re}(s)>0$. As shown by Callier and Winkin (1993) as well as Curtain and Zwart (1995), it is possible for a boundary control system to obtain a closed form expression for the transfer function by taking the Laplace transforms of the original partial differential equation and solving the resulting boundary value problem. This approach will be applied here to the analysed class of hyperbolic DPSs.

3.2. General transfer function matrix. After Laplace transform $\mathcal{L}_{t}$ with respect to the variable $t$, the general system (7) can be written as

$$
\frac{\partial x(l, s)}{\partial l}=\Lambda^{-1}(K-s I) x(l, s)+\Lambda^{-1} x(l, 0),
$$

where $x(l, 0)$ represents the initial conditions (12). Introducing, for the sake of brevity, the matrix $P(s)$ of the following form:

$$
P(s)=\Lambda^{-1}(K-s I),
$$

the general solution of (36) is given by

$$
x(l, s)=\Lambda^{-1} \int_{0}^{l} \mathrm{e}^{P(s)(l-\xi)} x(\xi, 0) \mathrm{d} \xi+\mathrm{e}^{P(s) l} x(0, s),
$$

where the first term on the right-hand side represents the response to the initial conditions at $t=0$ and the second the response to the boundary conditions at $l=0$.

Equation (38) can be identified with the state transition equation (34), except that the former is in the Laplace transform domain whereas the latter in the time domain. Moreover, the role of the input function $u(t)$ in (34) is played by the Laplace-transformed boundary conditions $x(0, s)$ in 38$)$. Therefore, assuming the case of the distributed output (29), the matrix $\mathrm{e}^{P(s) l}$ in (38), which is the resolvent of the operator $A$,

$$
R(s, A)=(s I-A)^{-1}=\mathrm{e}^{P(s) l} \text { for } \operatorname{Re}(s)>0,
$$

can be referred to as a general $n \times n$ transfer function matrix $G(l, s)$ of the system (7) with $n$ boundary inputs imposed at $l=0$ and the parameter $l$ indicating the spatial position for which the transfer function is to be evaluated.

In order to calculate the matrix exponential $\mathrm{e}^{P(s) l}$ in (39), we assume that $P(s)$ can be diagonalized as follows:

$$
P(s)=\Psi(s) \Phi(s) \Psi^{-1}(s),
$$

where $\Phi(s)$ is a diagonal matrix of eigenvalues of $P(s)$ and $\Psi(s)$ consists of its (column) eigenvectors. Consequently, Eqn. 38 can be written in the following form:

$$
x(l, s)=\Lambda^{-1} \int_{0}^{l} G(l-\xi, s) x(\xi, 0) \mathrm{d} \xi+G(l, s) x(0, s),
$$

where

$$
G(l, s)=\mathrm{e}^{P(s) l}=\Psi(s) \mathrm{e}^{\Phi(s) l} \Psi^{-1}(s) .
$$


3.3. Transfer function matrices of the secondorder system. In the case of the second-order system described by (15) and (16), one can distinguish two lumped input signals represented by the boundary conditions 17) or (18), and two output signals representing the distribution of the state variables $x_{1}(l, t)$ and $x_{2}(l, t)$ (see Eqn. (29)). For the purpose of further analysis, the following two definitions are introduced, based on the well-known notion of the transfer function as the ratio of the Laplace transform of the output signal to the Laplace transform of the input signal under the assumption that the other input signals as well as all initial conditions are equal to zero.

Definition 5. The transfer function matrix of the system described by Eqns. (15) and 16 is defined for the case of the congruent boundary inputs (17) as the matrix of the following form:

$$
G(l, s)=\left[\begin{array}{ll}
G_{11}(l, s) & G_{12}(l, s) \\
G_{21}(l, s) & G_{22}(l, s)
\end{array}\right],
$$

where

$$
G_{11}(l, s)=\frac{x_{1}(l, s)}{x_{1}(0, s)}, \quad G_{21}(l, s)=\frac{x_{2}(l, s)}{x_{1}(0, s)},
$$

for $x_{2}(0, s)=0$, and

$$
G_{12}(l, s)=\frac{x_{1}(l, s)}{x_{2}(0, s)}, \quad G_{22}(l, s)=\frac{x_{2}(l, s)}{x_{2}(0, s)},
$$

for $x_{1}(0, s)=0$, all for zero initial conditions, $x_{1}(l, 0)=$ $x_{2}(l, 0)=0$.

Definition 6. The transfer function matrix of the system described by Eqns. (15) and (16) is defined for the case of the incongruent boundary inputs $(18)$ as the matrix of the following form:

$$
\bar{G}(l, s)=\left[\begin{array}{ll}
\bar{G}_{11}(l, s) & \bar{G}_{12}(l, s) \\
\bar{G}_{21}(l, s) & \bar{G}_{22}(l, s)
\end{array}\right],
$$

where

$$
\bar{G}_{11}(l, s)=\frac{x_{1}(l, s)}{x_{1}(0, s)}, \quad \bar{G}_{21}(l, s)=\frac{x_{2}(l, s)}{x_{1}(0, s)},
$$

for $x_{2}(L, s)=0$, and

$$
\bar{G}_{12}(l, s)=\frac{x_{1}(l, s)}{x_{2}(L, s)}, \quad \bar{G}_{22}(l, s)=\frac{x_{2}(l, s)}{x_{2}(L, s)},
$$

for $x_{1}(0, s)=0$, all for zero initial conditions, $x_{1}(l, 0)=$ $x_{2}(l, 0)=0$.

The vector of Laplace transforms of the state variables

$$
x(l, s)=\left[\begin{array}{ll}
x_{1}(l, s) & x_{2}(l, s)
\end{array}\right]^{T}
$$

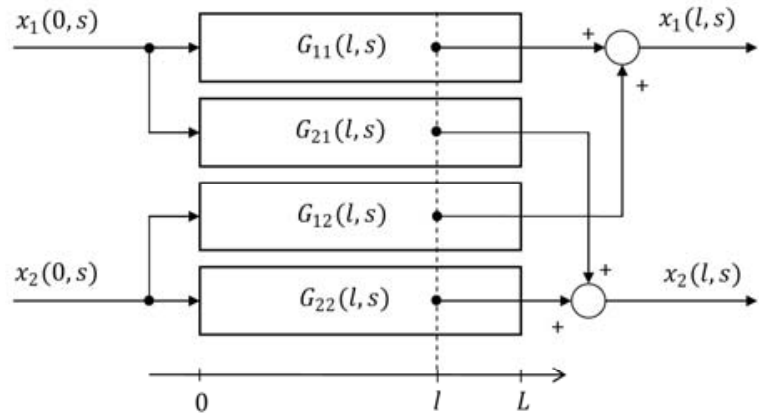

Fig. 1. Block diagram of the transfer function model for the congruent boundary inputs.

can therefore be determined for the congruent boundary inputs, assuming zero initial conditions, based on the following equation:

$$
x(l, s)=G(l, s) u(s)
$$

for $u(s)=\left[\begin{array}{ll}x_{1}(0, s) & x_{2}(0, s)\end{array}\right]^{T}$, which is obviously a particular case of (41). A block diagram of the transfer function model for the congruent boundary inputs is presented in Fig. 1 .

On the other hand, for the case of the incongruent boundary inputs (see Fig. 2), the formula for the vector of the state variables takes the following form:

$$
x(l, s)=\bar{G}(l, s) u(s)
$$

for $u(s)=\left[\begin{array}{ll}x_{1}(0, s) & x_{2}(L, s)\end{array}\right]^{T}$. Referring to the analysis presented in Section 3.2 the matrix $P(s)$ in 37) takes for the case considered the following form:

$$
P(s)=\left[\begin{array}{cc}
p_{11}(s) & p_{12} \\
p_{21} & p_{22}(s)
\end{array}\right]=\left[\begin{array}{cc}
\frac{k_{11}-s}{\lambda_{1}} & \frac{k_{12}}{\lambda_{1}} \\
\frac{k_{21}}{\lambda_{2}} & \frac{k_{22}-s}{\lambda_{2}}
\end{array}\right],
$$

and its eigendecomposition (40) results in the following matrix of eigenvalues:

$$
\Phi(s)=\operatorname{diag}\left(\phi_{1}(s), \phi_{2}(s)\right),
$$

with

$$
\phi_{1,2}(s)=\alpha(s) \pm \beta(s)
$$

where

$$
\alpha(s)=\frac{1}{2}\left(p_{11}(s)+p_{22}(s)\right)
$$

and

$$
\beta(s)=\frac{1}{2} \sqrt{\left(p_{11}(s)-p_{22}(s)\right)^{2}+4 p_{12} p_{21}} .
$$

Assuming as an example $k_{11}<0, k_{22}<0$ and $k_{12}=k_{21}=0$ in 15 and (16) for the case of the 


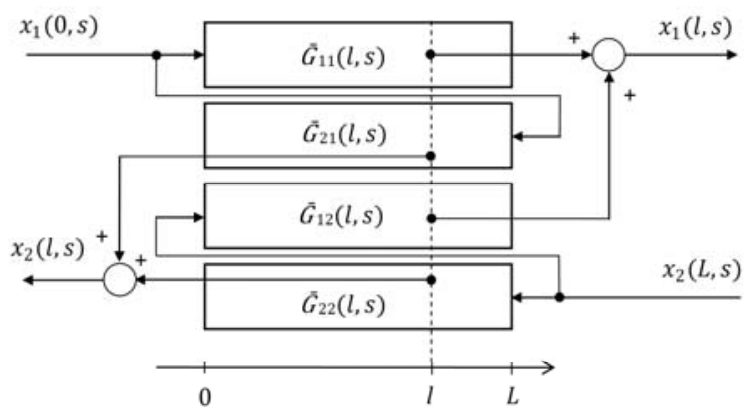

Fig. 2. Block diagram of the transfer function model for the incongruent boundary inputs.

congruent boundary inputs 177 , we immediately obtain, based on (52) and (42), the transfer function matrix $G(l, s)$ in the diagonal form,

$$
G(l, s)=\left[\begin{array}{cc}
\kappa_{1}(l) \mathrm{e}^{-s \tau_{1}(l)} & 0 \\
0 & \kappa_{2}(l) \mathrm{e}^{-s \tau_{2}(l)}
\end{array}\right],
$$

where

$$
\begin{aligned}
\kappa_{1}(l) & =\mathrm{e}^{\frac{k_{11}}{\lambda_{1}} l}, & \kappa_{2}(l) & =\mathrm{e}^{\frac{k_{22}}{\lambda_{2}} l}, \\
\tau_{1}(l) & =\frac{l}{\lambda_{1}}, & \tau_{2}(l) & =\frac{l}{\lambda_{2}} .
\end{aligned}
$$

The above representation should be considered a particular case of the two separate proportional-time-delay systems. Furthermore, assuming also $k_{11}=k_{22}=0$, we obtain two pure time-delay systems, $G_{11}(l, s)=\mathrm{e}^{-s \tau_{1}(l)}$ and $G_{22}(l, s)=\mathrm{e}^{-s \tau_{2}(l)}$.

In the next subsection the analytical closed form expressions for the individual elements of the transfer function matrices $G(l, s)$ and $\bar{G}(l, s)$ are derived for the general case of the non-zero parameters, taking into account the two different forms of the boundary conditions introduced in Section 2.3

\subsection{Analytical expressions for the transfer functions.}

Proposition 1. The transfer functions included in the matrix $G(l, s)$ in (43) take for the case of the congruent boundary inputs (17) the following form:

$$
\begin{aligned}
G_{11}(l, s) & =\frac{\phi_{1}(s)-p_{22}(s)}{\phi_{1}(s)-\phi_{2}(s)} \mathrm{e}^{\phi_{1}(s) l} \\
& -\frac{\phi_{2}(s)-p_{22}(s)}{\phi_{1}(s)-\phi_{2}(s)} \mathrm{e}^{\phi_{2}(s) l}, \\
G_{12}(l, s) & =\frac{p_{12}}{\phi_{1}(s)-\phi_{2}(s)}\left(\mathrm{e}^{\phi_{1}(s) l}-\mathrm{e}^{\phi_{2}(s) l}\right),
\end{aligned}
$$

$$
\begin{aligned}
G_{21}(l, s) & =\frac{p_{21}}{\phi_{1}(s)-\phi_{2}(s)}\left(\mathrm{e}^{\phi_{1}(s) l}-\mathrm{e}^{\phi_{2}(s) l}\right) \\
G_{22}(l, s) & =\frac{\phi_{1}(s)-p_{11}(s)}{\phi_{1}(s)-\phi_{2}(s)} \mathrm{e}^{\phi_{1}(s) l} \\
& -\frac{\phi_{2}(s)-p_{11}(s)}{\phi_{1}(s)-\phi_{2}(s)} \mathrm{e}^{\phi_{2}(s) l}
\end{aligned}
$$

where $p_{11}(s), p_{12}, p_{21}$ and $p_{22}(s)$ are elements of the matrix $P(s)$ in (52) and $\phi_{1}(s), \phi_{2}(s)$ are its eigenvalues given by (53)-(56).

Proof. By applying the Laplace transform $\mathcal{L}_{t}$ to 15 and (16), one obtains, assuming zero initial conditions $x_{1}(l, 0)=0$ and $x_{2}(l, 0)=0$, the following equations:

$$
\begin{aligned}
& \lambda_{1} \frac{\partial x_{1}(l, s)}{\partial l}=\left(k_{11}-s\right) x_{1}(l, s)+k_{12} x_{2}(l, s), \\
& \lambda_{2} \frac{\partial x_{2}(l, s)}{\partial l}=k_{21} x_{1}(l, s)+\left(k_{22}-s\right) x_{2}(l, s) .
\end{aligned}
$$

Taking the Laplace transform again, now with respect to the spatial variable $l$,

$$
x(q, s)=\mathcal{L}_{l}\{x(l, s)\}
$$

and taking into account that

$$
\mathcal{L}_{l}\left\{\frac{\partial x(l, s)}{\partial l}\right\}=q x(q, s)-x(0, s)
$$

transforms Eqns. (64) and 65) into the following form:

$$
\begin{aligned}
& x_{1}(q, s)=\frac{q-\frac{k_{22}-s}{\lambda_{2}}}{M(q, s)} x_{1}(0, s)+\frac{\frac{k_{12}}{\lambda_{1}}}{M(q, s)} x_{2}(0, s), \\
& x_{2}(q, s)=\frac{\frac{k_{21}}{\lambda_{2}}}{M(q, s)} x_{1}(0, s)+\frac{q-\frac{k_{11}-s}{\lambda_{1}}}{M(q, s)} x_{2}(0, s),
\end{aligned}
$$

where $M(q, s)$ is the characteristic polynomial of the matrix $P(s)$

$$
\begin{aligned}
M(q, s) & =\operatorname{det}(q I-P(s))= \\
& =\left(q-\phi_{1}(s)\right)\left(q-\phi_{2}(s)\right) .
\end{aligned}
$$

Finding the inverse Laplace transform of 68 and (69) with respect to $q$ by taking advantage of the following property (Friedly, 1972):

$$
\mathcal{L}_{q}^{-1}\left\{\frac{S(q)}{T(q)}=\frac{S(q)}{\prod_{j=1}^{N}\left(q-\lambda_{j}\right)}\right\}=\sum_{j=1}^{N} \frac{S\left(\lambda_{j}\right)}{\left.\frac{\mathrm{d} T(q)}{\mathrm{d} q}\right|_{q=\lambda_{j}}} \mathrm{e}^{\lambda_{j} t},
$$


where $S(q)$ and $T(q)$ represent polynomials in $q$ of degree $M$ and $N>M$, respectively, and $\lambda_{j}$ is a single root of $T(q)$, yields the following form of the equations:

$$
\begin{aligned}
& x_{1}(l, s)=G_{11}(l, s) x_{1}(0, s)+G_{12}(l, s) x_{2}(0, s), \\
& x_{2}(l, s)=G_{21}(l, s) x_{1}(0, s)+G_{22}(l, s) x_{2}(0, s),
\end{aligned}
$$

where the expressions on $G_{11}(l, s), G_{12}(l, s), G_{21}(l, s)$ and $G_{22}(l, s)$ are given by (60)- 63 .

Lemma 1. For any $x, y, z \in \mathbb{C}$ such that $z \neq 0$ and $z \neq y$, the following identity holds:

$$
\mathrm{e}^{x}-\frac{y}{z} \mathrm{e}^{-x}=\frac{z-y}{z}\left(\cosh x+\frac{z+y}{z-y} \sinh x\right) .
$$

Proof. By using the well-known identities

$$
\sinh x=\frac{\mathrm{e}^{x}-\mathrm{e}^{-x}}{2}
$$

and

$$
\cosh x=\frac{\mathrm{e}^{x}+\mathrm{e}^{-x}}{2},
$$

the right-hand side of (74) can be transformed in the following way:

$$
\begin{aligned}
& \frac{z-y}{z}\left(\cosh x+\frac{z+y}{z-y} \sinh x\right) \\
& =\frac{z-y}{2 z}\left(\mathrm{e}^{x}+\mathrm{e}^{-x}+\frac{z+y}{z-y}\left(\mathrm{e}^{x}-\mathrm{e}^{-x}\right)\right) \\
& =\frac{z-y}{2 z}\left(\frac{2 z}{z-y} \mathrm{e}^{x}-\frac{2 y}{z-y} \mathrm{e}^{-x}\right) \\
& =\mathrm{e}^{x}-\frac{y}{z} \mathrm{e}^{-x} .
\end{aligned}
$$

Proposition 2. The transfer functions (60)-63) can be expressed in the following equivalent form using the hyperbolic functions:

$$
\begin{aligned}
G_{11}(l, s)= & \mathrm{e}^{\alpha(s) l}(\cosh \beta(s) l \\
& \left.+\frac{\alpha(s)-p_{22}(s)}{\beta(s)} \sinh \beta(s) l\right), \\
G_{12}(l, s)= & \frac{p_{12}}{\beta(s)} \mathrm{e}^{\alpha(s) l} \sinh \beta(s) l, \\
G_{21}(l, s)= & \frac{p_{21}}{\beta(s)} \mathrm{e}^{\alpha(s) l} \sinh \beta(s) l, \\
G_{22}(l, s)= & \mathrm{e}^{\alpha(s) l}(\cosh \beta(s) l \\
& \left.+\frac{\alpha(s)-p_{11}(s)}{\beta(s)} \sinh \beta(s) l\right),
\end{aligned}
$$

where $\alpha(s)$ and $\beta(s)$ are given by (55) and (56).
Proof. The transfer function $G_{11}(l, s)$ described by (60) can be transformed using (54) and Lemma 1 into the following form:

$$
\begin{aligned}
& G_{11}(l, s) \\
&=\frac{\phi_{1}(s)}{\phi_{1}(s)-\phi_{2}(s)} \mathrm{e}^{\alpha(s) l} \mathrm{e}^{\beta(s) l} \\
&-\frac{p_{22}(s)}{\phi_{1}(s)-\phi_{2}(s)} \mathrm{e}^{\alpha(s) l} \mathrm{e}^{\beta(s) l} \\
&-\frac{\phi_{2}(s)}{\phi_{1}(s)-\phi_{2}(s)} \mathrm{e}^{\alpha(s) l} \mathrm{e}^{-\beta(s) l} \\
&+\frac{p_{22}(s)}{\phi_{1}(s)-\phi_{2}(s)} \mathrm{e}^{\alpha(s) l} \mathrm{e}^{-\beta(s) l} \\
&= \frac{\phi_{1}(s)}{\phi_{1}(s)-\phi_{2}(s)} \mathrm{e}^{\alpha(s) l}\left(\mathrm{e}^{\beta(s) l}-\frac{\phi_{2}(s)}{\phi_{1}(s)}(s) \mathrm{e}^{-\beta(s) l}\right) \\
&-\frac{p_{22}(s)}{\phi_{1}(s)-\phi_{2}(s)} \mathrm{e}^{\alpha(s) l}\left(\mathrm{e}^{\beta(s) l}-\mathrm{e}^{-\beta(s) l}\right) \\
&= \mathrm{e}^{\alpha(s) l}\left(\cosh \beta(s) l+\frac{\phi_{1}(s)+\phi_{2}(s)}{\phi_{1}(s)-\phi_{2}(s)} \sinh \beta(s) l\right) \\
&-2 \frac{p_{22}(s)}{\phi_{1}(s)-\phi_{2}(s)} \mathrm{e}^{\alpha(s) l} \sinh \beta(s) l
\end{aligned}
$$

Then, by making the substitutions $\phi_{1}(s)-\phi_{2}(s)=$ $2 \beta(s)$ and $\phi_{1}(s)+\phi_{2}(s)=2 \alpha(s)$, one obtains the hyperbolic form (78) of $G_{11}(l, s)$. Due to the obvious symmetry, one can similarly obtain the hyperbolic version (81) of $G_{22}(l, s)$. On the other hand, for the transfer function $G_{12}(l, s)$ we have

$$
\begin{aligned}
G_{12}(l, s) & =\frac{p_{12}}{\phi_{1}(s)-\phi_{2}(s)} \mathrm{e}^{\alpha(s) l}\left(\mathrm{e}^{\beta(s) l}-\mathrm{e}^{-\beta(s) l}\right) \\
& =2 \frac{p_{12}}{\phi_{1}(s)-\phi_{2}(s)} \mathrm{e}^{\alpha(s) l} \sinh \beta(s) l \\
& =\frac{p_{12}}{\beta(s)} \mathrm{e}^{\alpha(s) l} \sinh \beta(s) l,
\end{aligned}
$$

and, in a similar fashion, we come to the hyperbolic form (80) of $G_{21}(l, s)$.

Remark 5. By making in the transfer functions 60 -63 the substitution $l=0$, one obtains $\mathrm{e}^{\phi_{1}(s) l}=\mathrm{e}^{\phi_{2}(s) l}=1$ and finally $G_{11}(0, s)=1, G_{12}(0, s)=0, G_{21}(0, s)=0$, $G_{22}(0, s)=1$. This result means that for $l=0$, a given output variable $x_{i}(l, t)$ is identically equal to the relevant boundary input $u_{i}(t)$, without any cross-interactions from the second boundary input (see Fig. 11). Analogous results can be obtained based on the analysis of the hyperbolic form (78)- 81 of the transfer functions. In this case one obtains, for $l=0, \mathrm{e}^{\alpha(s) l}=1, \sinh \beta(s) l=0$ and $\cosh \beta(s) l=1$, which leads to the same values of the transfer functions. 
Proposition 3. The transfer functions included in the matrix $\bar{G}(l, s)$ in (46) for the case of the incongruent boundary inputs (18) take the following form:

$$
\begin{aligned}
& \bar{G}_{11}(l, s) \\
& =\frac{\mathrm{e}^{\phi_{2}(s) L} \mathrm{e}^{\phi_{1}(s) l}\left(\phi_{1}(s)-p_{22}(s)\right)}{\mathrm{e}^{\phi_{2}(s) L}\left(\phi_{1}(s)-p_{22}(s)\right)-\mathrm{e}^{\phi_{1}(s) L}\left(\phi_{2}(s)-p_{22}(s)\right)} \\
& -\frac{\mathrm{e}^{\phi_{1}(s) L} \mathrm{e}^{\phi_{2}(s) l}\left(\phi_{2}(s)-p_{22}(s)\right)}{\mathrm{e}^{\phi_{2}(s) L}\left(\phi_{1}(s)-p_{22}(s)\right)-\mathrm{e}^{\phi_{1}(s) L}\left(\phi_{2}(s)-p_{22}(s)\right)},
\end{aligned}
$$

$\bar{G}_{12}(l, s)$

$=\frac{p_{12}\left(\mathrm{e}^{\phi_{2}(s) l}-\mathrm{e}^{\phi_{1}(s) l}\right)}{\mathrm{e}^{\phi_{2}(s) L}\left(\phi_{2}(s)-p_{11}(s)\right)-\mathrm{e}^{\phi_{1}(s) L}\left(\phi_{1}(s)-p_{11}(s)\right)}$,

$$
\begin{aligned}
& \bar{G}_{21}(l, s) \\
& =\frac{p_{21}\left(\mathrm{e}^{\phi_{2}(s) L} \mathrm{e}^{\phi_{1}(s) l}-\mathrm{e}^{\phi_{1}(s) L} \mathrm{e}^{\phi_{2}(s) l}\right)}{\mathrm{e}^{\phi_{2}(s) L}\left(\phi_{1}(s)-p_{22}(s)\right)-\mathrm{e}^{\phi_{1}(s) L}\left(\phi_{2}(s)-p_{22}(s)\right)},
\end{aligned}
$$

$$
\begin{aligned}
& \bar{G}_{22}(l, s) \\
& =\frac{\mathrm{e}^{\phi_{2}(s) l}\left(\phi_{2}(s)-p_{11}(s)\right)-\mathrm{e}^{\phi_{1}(s) l}\left(\phi_{1}(s)-p_{11}(s)\right)}{\mathrm{e}^{\phi_{2}(s) L}\left(\phi_{2}(s)-p_{11}(s)\right)-\mathrm{e}^{\phi_{1}(s) L}\left(\phi_{1}(s)-p_{11}(s)\right)},
\end{aligned}
$$

where $p_{11}(s), p_{12}, p_{21}$ and $p_{22}(s)$ are elements of the matrix $P(s)$ in (52) and $\phi_{1}(s), \phi_{2}(s)$ are its eigenvalues given by (53)- (56).

Proof. The solution of the set of the differential equations (64) and 65), now with the incongruent boundary conditions (see Definition 3), is given in the following form:

$$
\begin{aligned}
& x_{1}(l, s)=\bar{G}_{11}(l, s) x_{1}(0, s)+\bar{G}_{12}(l, s) x_{2}(L, s), \\
& x_{2}(l, s)=\bar{G}_{21}(l, s) x_{1}(0, s)+\bar{G}_{22}(l, s) x_{2}(L, s),
\end{aligned}
$$

where the expressions for $\bar{G}_{11}(l, s), \bar{G}_{12}(l, s), \bar{G}_{21}(l, s)$ and $\bar{G}_{22}(l, s)$ are given by (84)- 87$)$.

Proposition 4. The transfer functions 84-87) can be expressed in the following equivalent form using the hyperbolic functions:

$$
\begin{aligned}
& \bar{G}_{11}(l, s) \\
& =\frac{\mathrm{e}^{\alpha(s) l} \beta(s) \cosh \beta(s)(l-L)}{\beta(s) \cosh \beta(s) L-\left(\alpha(s)-p_{22}(s)\right) \sinh \beta(s) L} \\
& +\frac{\mathrm{e}^{\alpha(s) l}\left(\alpha(s)-p_{22}(s)\right) \sinh \beta(s)(l-L)}{\beta(s) \cosh \beta(s) L-\left(\alpha(s)-p_{22}(s)\right) \sinh \beta(s) L},
\end{aligned}
$$

$$
\begin{aligned}
& \bar{G}_{12}(l, s) \\
& =\frac{p_{12} \mathrm{e}^{\alpha(s)(l-L)} \sinh \beta(s)(l)}{\beta(s) \cosh \beta(s) L+\left(\alpha(s)-p_{11}(s)\right) \sinh \beta(s) L},
\end{aligned}
$$

$$
\begin{aligned}
& \bar{G}_{21}(l, s) \\
& =\frac{p_{21} \mathrm{e}^{\alpha(s) l} \sinh \beta(s)(l-L)}{\beta(s) \cosh \beta(s) L-\left(\alpha(s)-p_{22}(s)\right) \sinh \beta(s) L},
\end{aligned}
$$

$$
\begin{aligned}
& \bar{G}_{22}(l, s) \\
& =\frac{\mathrm{e}^{\alpha(s)(l-L)} \beta(s) \cosh \beta(s) l}{\beta(s) \cosh \beta(s) L+\left(\alpha(s)-p_{11}(s)\right) \sinh \beta(s) L} \\
& +\frac{\mathrm{e}^{\alpha(s)(l-L)}\left(\alpha(s)-p_{11}(s)\right) \sinh \beta(s) l}{\beta(s) \cosh \beta(s) L+\left(\alpha(s)-p_{11}(s)\right) \sinh \beta(s) L} .
\end{aligned}
$$

Proof. The result is derived as in the case of the proof of Result 2] i.e., using (54) and Lemma1.

Remark 6. In the case of the incongruent boundary inputs one obtains $\bar{G}_{11}(0, s)=1, \bar{G}_{12}(0, s)=0$, $\bar{G}_{21}(L, s)=0$ and $\bar{G}_{22}(L, s)=1$ (see Fig. 2).

3.5. Pole-zero analysis. The pole-zero analysis can be started with the simplified form of the analysed transfer functions, discussed at the end of Section 3.3, i.e., for $k_{12}=k_{21}=0$. In this case we obtain $\phi_{1}(s)=p_{11}(s)$, $\phi_{2}(s)=p_{22}(s)$ and the transfer functions are given by the matrix (57) with $G_{12}(l, s)=G_{21}(l, s)=0$ and $G_{11}(l, s), G_{22}(l, s)$ in the pure exponential form having neither poles nor zeros. Similar results can be obtained for the case of the incongruent inputs.

Considering the more general case of the non-zero parameters, one can suppose that the poles of the transfer functions (60) - 63) and 840 -87) occur when

$$
\phi_{1}(s)=\phi_{2}(s)
$$

with

$$
s_{1,2}=\frac{k_{22} \lambda_{1}-k_{11} \lambda_{2}}{\lambda_{1}-\lambda_{2}} \pm \frac{2 \sqrt{-k_{12} k_{21} \lambda_{1} \lambda_{2}}}{\lambda_{1}-\lambda_{2}} .
$$

However, Eqn. 94 expresses also the condition for "zeroing" all transfer function numerators. Thus, since the poles and zeros are defined for the reduced form of the transfer function, we can state that all the transfer functions considered have no poles. Furthermore, transfer functions $G_{11}(l, s), G_{22}(l, s)$ and their incongruent counterparts have no zeros either. The above results can be regarded as a generalization of the results obtained for 
the simplified form of the transfer functions considered in Section 3.3 .

Slightly different is the case of the "crossover" channels $G_{12}(l, s)$ and $G_{21}(l, s)$, for which the condition for the occurrence of zeros is

$$
\mathrm{e}^{\phi_{1}(s) l}=\mathrm{e}^{\phi_{2}(s) l} .
$$

Thus, due to the periodicity of the exponential function along the imaginary axis, this condition means that the transfer functions considered have infinitely many zeros as stated in the following result.

Proposition 5. The transfer functions $G_{12}(l, s)$, $G_{21}(l, s)$ and $\bar{G}_{12}(l, s)$ have infinitely many zeros of the following form:

$$
\begin{aligned}
z_{1,2}^{(k)}(l) & =\frac{k_{22} \lambda_{1}-k_{11} \lambda_{2}}{\left(\lambda_{1}-\lambda_{2}\right)} \pm \\
& \pm \frac{2 \sqrt{-\lambda_{1}^{2} \lambda_{2}^{2} k^{2} \pi^{2}-\lambda_{1} \lambda_{2} k_{12} k_{21} l^{2}}}{\left(\lambda_{1}-\lambda_{2}\right) l}
\end{aligned}
$$

for $k \in \mathbb{N}$.

Proof. Based on (79), 80, and (91), one can observe that the zeros of these transfer functions are given as the roots of the following equation:

$$
\sinh \beta(s) l=0,
$$

which is satisfied for

$$
\beta(s) l=k \pi i, \quad k \in \mathbb{Z} .
$$

Taking into account (56), Eqn. (99) can be transformed to

$$
\left(p_{11}(s)-p_{22}(s)\right)^{2}+4 p_{12} p_{21}=-\frac{4 k^{2} \pi^{2}}{l^{2}} .
$$

Using the relationships in (52) and finding the solution of (100) in terms of $s$ gives as a result the expression (97).

Remark 7. The zeros $z_{1,2}^{(0)}(l)$ for $k=0$ in (97) are canceled by the poles $s_{1,2}$ in 95 , which is easy to show by inserting $k=0$ into (97) and comparing to (95).

Result 1. Taking into account (92), the zeros of the transfer function $\bar{G}_{21}(l, s)$ should satisfy the condition $\sinh \beta(s)(l-L)=0$. Thus, in order to determine their values, one should replace in 97) the variable $l$ with $l-L$.

3.6. Frequency responses. Based on the knowledge of the transfer functions it is possible to determine the frequency responses of the individual channels of the system under consideration. For this purpose, one should replace in the relationships 600 -63) and (84) in their hyperbolic equivalents (78)- 81 and $90-$ - 93 , the operator variable $s$ with the expression $i \omega$, where $\omega$ is the angular frequency. As a result, one obtains the corresponding frequency responses $G_{i j}(l, i \omega)$ of the channel connecting the $i$-th output to the $j$-th input, $i, j=$ 1,2 .

The graphical representation of these responses can take the form of three-dimensional graphs, taking into account the dependence of the frequency response on both the angular frequency $\omega$ and the spatial variable $l$. Another possibility is the representation in the form of the classical two-dimensional plots, determined for the fixed value of the spatial variable (Jovanović and Bamieh, 2006; Bartecki, 2007; 2009; Litrico and Fromion, 2009a). Considering as an example the Bode plot of the frequency response, the expressions for the logarithmic gain and phase take the following form:

$$
L_{i j}(l, \omega)=20 \log \left|G_{i j}(l, i \omega)\right|
$$

and

$$
\varphi_{i j}(l, \omega)=\arg \left[G_{i j}(l, i \omega)\right],
$$

where the expressions for the modulus and argument of the frequency response are as follows:

$$
\left|G_{i j}(l, i \omega)\right|=\sqrt{\operatorname{Re}^{2}\left\{G_{i j}(l, i \omega)\right\}+\operatorname{Im}^{2}\left\{G_{i j}(l, i \omega)\right\}}
$$

and

$$
\arg \left[G_{i j}(l, i \omega)\right]=\operatorname{atan} \frac{\operatorname{Im}\left\{G_{i j}(l, i \omega)\right\}}{\operatorname{Re}\left\{G_{i j}(l, i \omega)\right\}} .
$$

\section{Examples}

For a practical illustration of the above discussed issues, this section performs a transfer function analysis of a shell and tube heat exchanger, which can be considered a typical DPS whose mathematical description, after some assumptions, takes the form of Eqns. (15) and (16). The analysis is performed for both the exchanger operating in the parallel-flow mode, for which the boundary conditions have the form specified in Definition 2] and for the countercurrent-flow mode, with boundary conditions of the form adopted in Definition 3 Having derived, based on Eqns. (60)-63 and (84), the transfer functions of the exchanger, the pole-zero analysis is performed. Selected frequency responses for the parallel and countercurrent flow modes are also presented, both in the form of three-dimensional graphs as well as the classical two-dimensional Bode and Nyquist plots.

4.1. Parallel-flow heat exchanger. Under some simplifying assumptions, the dynamic properties of a shell and tube heat exchanger can be described, based on the thermal energy balance equations, by the following PDE system (Bartecki and Rojek, 2005; Bartecki, 2007; 
Delnero et al., 2004; Gvozdenac, 1990; Zavala-Río et al., 2009; Maidi et al., 2010):

$$
\begin{aligned}
& \frac{\partial \vartheta_{1}(l, t)}{\partial t}+v_{1} \frac{\partial \vartheta_{1}(l, t)}{\partial l}=\alpha_{1}\left(\vartheta_{2}(l, t)-\vartheta_{1}(l, t)\right), \\
& \frac{\partial \vartheta_{2}(l, t)}{\partial t}+v_{2} \frac{\partial \vartheta_{2}(l, t)}{\partial l}=\alpha_{2}\left(\vartheta_{1}(l, t)-\vartheta_{2}(l, t)\right),
\end{aligned}
$$

where the 1- and 2- sub-indexed figures represent the tube-side and shell-side fluid variables/coefficients, respectively; specifically, $\vartheta_{1}(l, t)$ and $\vartheta_{2}(l, t)$ are the temperature variations, $v_{1}$ and $v_{2}$ are the velocities, $\alpha_{1}$ and $\alpha_{2}$ are the heat transfer coefficients.

Referring Eqns. (105) and (106) to Eqn. (1), one obtains the following vector of the state variables:

$$
w(l, t)=\left[\vartheta_{1}(l, t) \quad \vartheta_{2}(l, t)\right]^{T},
$$

and the following matrices of constant coefficients:

$$
E=\left[\begin{array}{ll}
1 & 0 \\
0 & 1
\end{array}\right], \quad F=\left[\begin{array}{cc}
v_{1} & 0 \\
0 & v_{2}
\end{array}\right], \quad W=\left[\begin{array}{cc}
-\alpha_{1} & \alpha_{1} \\
\alpha_{2} & -\alpha_{2}
\end{array}\right] .
$$

Since $F E^{-1}$ is diagonal, Eqns. 105) and (106) are decoupled in the differential part and the diagonalization procedure described by Eqns. (3)-8) is not necessary. Assuming $v_{1}=1 \mathrm{~m} \cdot \mathrm{s}^{-1}, v_{2}=0.2 \mathrm{~m} \cdot \mathrm{s}^{-1}, \alpha_{1}=\alpha_{2}=$ $0.05 \mathrm{~s}^{-1}$ in 105 and (106), one obtains the following matrices of the system (7):

$$
\begin{gathered}
\Lambda=F=\left[\begin{array}{cc}
1 & 0 \\
0 & 0.2
\end{array}\right], \\
K=W=\left[\begin{array}{cc}
-0.05 & 0.05 \\
0.05 & -0.05
\end{array}\right],
\end{gathered}
$$

with $\lambda_{1}=v_{1}=1, \lambda_{2}=v_{2}=0.2$ being the eigenvalues of the system, and $K$ symmetric and negative semi-definite, which makes the system dissipative.

The fluid inlet temperatures $\vartheta_{1 i}, \vartheta_{2 i}$ can be taken as the input signals, which in the given case of the parallel-flow corresponds the following congruent boundary conditions:

$$
\begin{aligned}
& \vartheta_{1}(0, t)=\vartheta_{1 i}(t), \\
& \vartheta_{2}(0, t)=\vartheta_{2 i}(t) .
\end{aligned}
$$

The transfer functions of the heat exchanger are given by Eqns. 607 -63 or, equivalently, by (78) and represent the ratio of the Laplace transform of the fluid temperature along the spatial axis to the Laplace transform of the fluid temperature in the inlet section of the exchanger, i.e., for $l=0$ :

$$
\begin{aligned}
& \vartheta_{1}(l, s)=G_{11}(l, s) \vartheta_{1}(0, s)+G_{12}(l, s) \vartheta_{2}(0, s), \\
& \vartheta_{2}(l, s)=G_{21}(l, s) \vartheta_{1}(0, s)+G_{22}(l, s) \vartheta_{2}(0, s),
\end{aligned}
$$

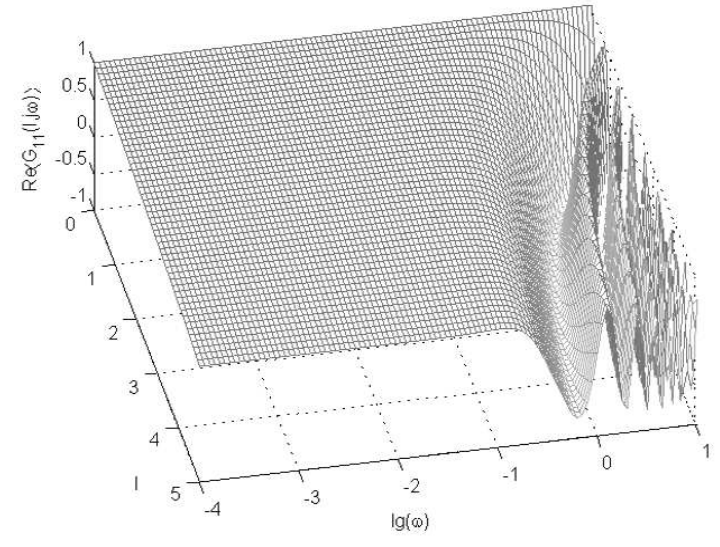

Fig. 3. Real part of the frequency response function $G_{11}(l, i \omega)$ for the parallel-flow heat exchanger.

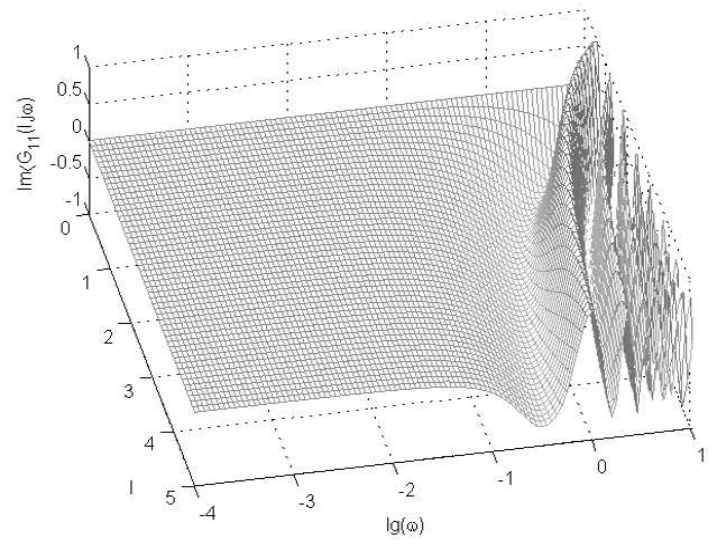

Fig. 4. Imaginary part of the frequency response function $G_{11}(l, i \omega)$ for the parallel-flow heat exchanger.

where $\vartheta_{i}(l, s)=\mathcal{L}_{t}\left\{\vartheta_{i}(l, t)\right\}$ is the Laplace transform of the function representing the distribution of the temperature of the $i$-th fluid, $i=1,2$.

According to the results presented in Section 3.5, all the transfer functions of the heat exchanger considered have no poles. Additionally, transfer functions $G_{11}(l, s)$ and $G_{22}(l, s)$ have no zeros either. On the other hand, for the transfer functions $G_{12}(L, s), G_{21}(L, s)$ and parameter values (109)-110) we obtain, based on Proposition 5 , infinitely many pairs of zeros of the following form:

$$
\begin{aligned}
& z_{1,2}^{(1)} \approx-0.0289 \pm 0.3187 i \\
& z_{1,2}^{(2)} \approx-0.0289 \pm 0.6306 i
\end{aligned}
$$

etc.

Figures 3 and 4 show three-dimensional graphs of the real and imaginary parts of the frequency response $G_{11}(l, i \omega)$ of the exchanger operating in the parallel-flow mode, determined based on 60 for the assumed 

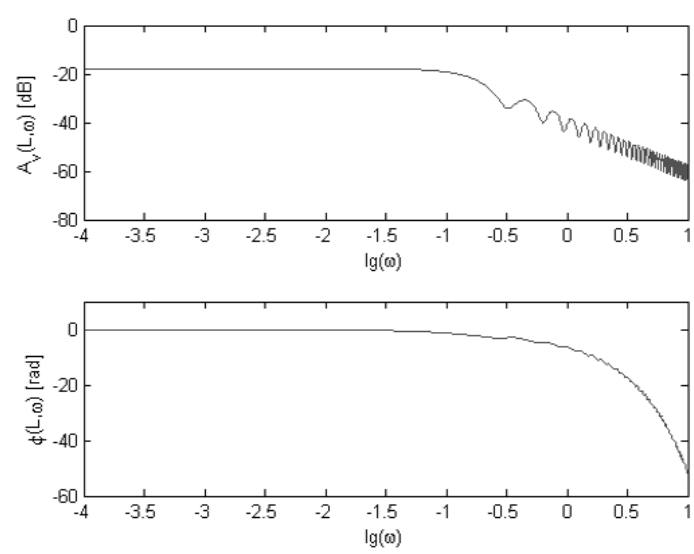

Fig. 5. Bode plot of the frequency response $G_{12}(L, i \omega)$ for the parallel-flow heat exchanger.

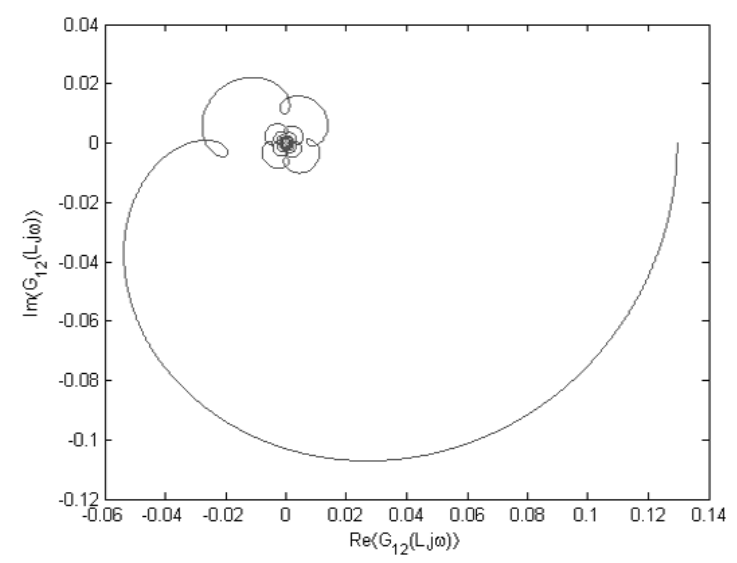

Fig. 6. Nyquist plot of the frequency response $G_{12}(L, i \omega)$ for the parallel-flow heat exchanger.

parameter values (109) and (110). One can observe characteristic oscillations caused by the aforementioned periodicity of the exponential function for the imaginary argument.

Next, Fig. 5 shows classical, two-dimensional Bode plots of the heat exchanger frequency responses $G_{12}(l, s)$, determined for $l=L$ based on Eqns. 61) and (101)-(104). The Nyquist plot for the same transfer function channel is presented in Fig. 6 As seen from the Bode plot, the increase in the frequency of the sinusoidal input signal initially causes a decrease in the amplitude of the output signal, and then it gives rise to a local maximum. To a lesser extent it affects the phase characteristics. As the frequency increases, the phenomenon repeats itself, which can be also observed as characteristic "loops" on the Nyquist plot. These oscillations are closely associated with the wave phenomena taking place inside the exchanger pipes (Friedly, 1972). On can notice a relationship between the zeros of the transfer functions $G_{12}(l, s)$ and $G_{21}(l, s)$ and the shape of the corresponding frequency responses. For example, the imaginary parts of the zeros $z^{(k)}=\alpha+\omega_{k} i$ calculated for $k=1,2$ in (115) and (116) correspond to the frequency values $\omega_{k}$ for which the local minima occur on the amplitude plot in Fig. 5

4.2. Countercurrent-flow heat exchanger. In the countercurrent mode of operation, the fluids involved in the heat exchange enter the exchanger from its opposite ends. The PDEs describing the dynamics of the heat exchanger have the same form (105)-(106) as for the parallel-flow mode, and the difference in the mathematical description consists in the opposite signs of fluid velocities $\left(v_{1}>0, v_{2}<0\right)$ as well as in the different boundary conditions

$$
\begin{aligned}
& \vartheta_{1}(0, t)=\vartheta_{1 i}(t), \\
& \vartheta_{2}(L, t)=\vartheta_{2 i}(t) .
\end{aligned}
$$

This situation represents the case of the incongruent boundary inputs (18). Assuming the parameter values (110) and the following values of fluid velocities: $v_{1}=$ $1 \mathrm{~m} \cdot \mathrm{s}^{-1}, v_{2}=-0.2 \mathrm{~m} \cdot \mathrm{s}^{-1}$, one obtains, based on (97), the following values of the zeros of the transfer function $\bar{G}_{12}(L, s)$ :

$$
\begin{aligned}
& z_{1,2}^{(1)} \approx-0.0406 \pm 0.2064 i, \\
& z_{1,2}^{(2)} \approx-0.0406 \pm 0.4174 i,
\end{aligned}
$$

etc., and the same values for the zeros of the transfer function $\bar{G}_{21}(0, s)$.

Figures 7 and 8 show the three-dimensional graphs of the real and the imaginary parts of the frequency response $\bar{G}_{21}(l, i \omega)$ of the exchanger operating in the countercurrent-flow mode. Unlike in the case of the parallel mode, due to the reversal of the flow direction for the second fluid, the largest amplitude of the oscillations occurs now at $l=0$. Figure 9 shows classical Bode plots of the heat exchanger frequency responses $\bar{G}_{22}(l, s)$, determined based on Eqns. (87) and 101-104 for the outlet point of the the second fluid, i.e., for $l=0$. The Nyquist plot of the same frequency response is shown in Fig. 10

An analysis of the frequency responses of the heat exchanger exhibits typical characteristics of systems with distributed delay (Górecki et al., 1989). In particular, in the case of the "straightforward" transfer functions $G_{11}(l, s), G_{22}(l, s)$ and their incongruent counterparts, one can notice the dominant influence of the transport delay in the fluid flow. The amplitude damping of the sinusoidal oscillations in the real and imaginary parts of the frequency response is relatively small, which is reflected in the circular-shaped Nyquist plot, similar to the one of the pure delay system (see Figs. 9 and 10). 


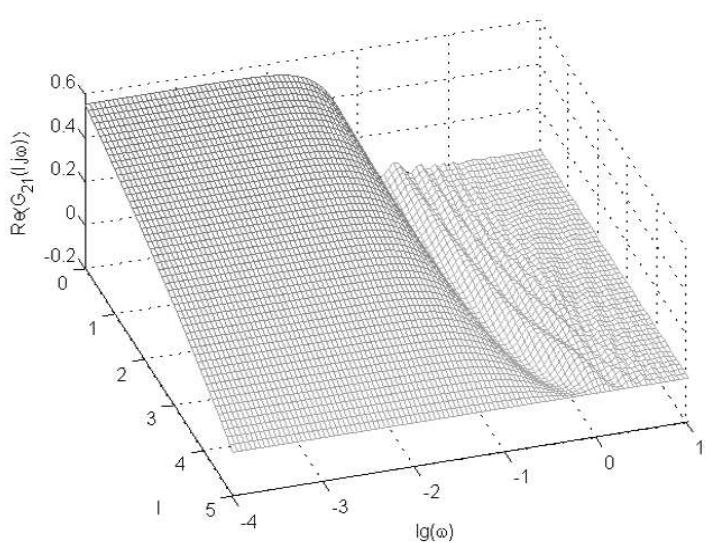

Fig. 7. Real part of the frequency response function $\bar{G}_{21}(l, i \omega)$ for the countercurrent-flow heat exchanger.

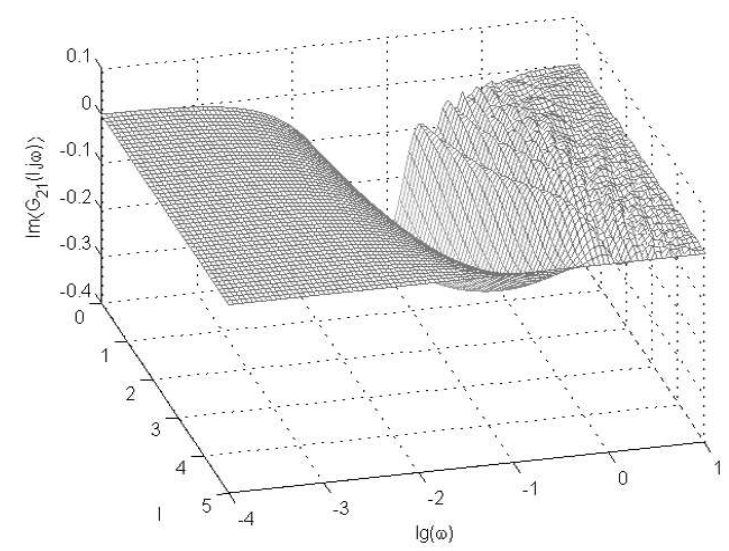

Fig. 8. Imaginary part of the frequency response function $\bar{G}_{21}(l, i \omega)$ for the countercurrent-flow heat exchanger.

On the other hand, in the case of the "crossover" transfer functions $G_{12}(l, s)$ and $G_{21}(l, s)$, the damping of the input signal with increasing frequency is much greater, as for the "straight-forward" channels (see Figs. 5]and 6).

\section{Conclusion}

In the article, a general analytic form of the transfer functions for a class of DPSs of hyperbolic type with two boundary inputs and two distributed outputs has been derived and analyzed. As shown here, taking into account the spatio-temporal dynamics of these systems significantly affects their transfer function representation. Unlike in the case of lumped systems, the transfer functions derived for the DPS contain irrational functions, e.g., exponential and/or hyperbolic ones. The "non-standard" form of these transfer functions causes some peculiarities which do not appear in the rational-form case, such as an infinite number of poles
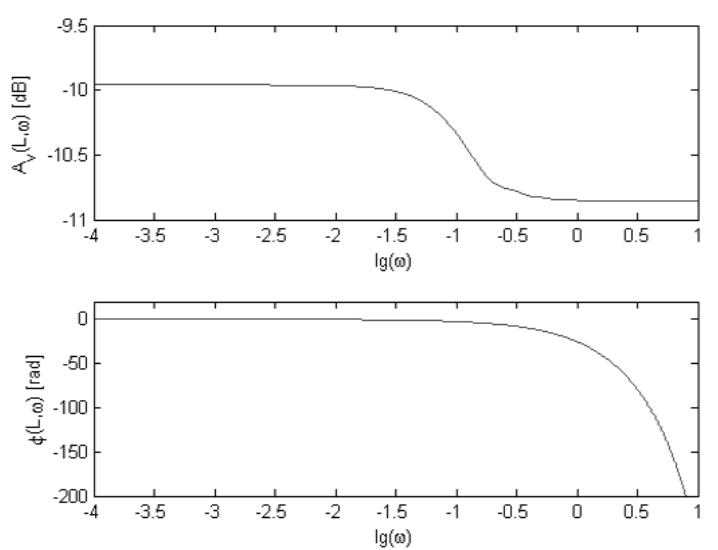

Fig. 9. Bode plot of the frequency response $\bar{G}_{22}(0, i \omega)$ for the countercurrent-flow heat exchanger.

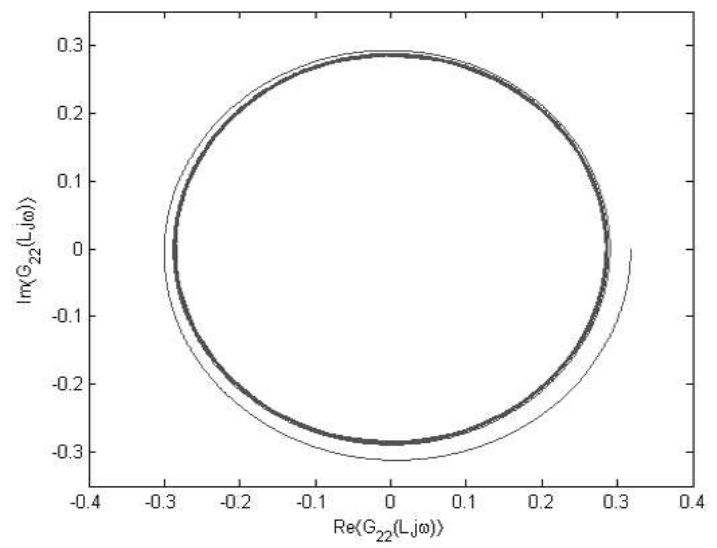

Fig. 10. Nyquist plot of the frequency response $\bar{G}_{22}(0, i \omega)$ for the countercurrent-flow heat exchanger.

and/or zeros. Also the spatial location of the input signals, represented by the appropriate boundary conditions, significantly influences the form of the transfer functions. Two kinds of Dirichlet boundary conditions have been examined: congruent, where both inputs are given at the same point of the spatial domain, and incongruent, where each input is located in a different point of the domain.

The results of the transfer function analysis have been illustrated on the example of a shell and tube heat exchanger operating in parallel- and countercurrent-flow modes, considered to be a typical example of a hyperbolic DPS with congruent and incongruent boundary inputs, respectively. Based on the transfer function representation, the zero-pole analysis has been performed and the selected frequency responses of the exchanger have been shown. They have been presented both in the form of three-dimensional graphs, taking into account the spatio-temporal specificity of the system, and as the classical two-dimensional Bode and Nyquist plots derived 
for a fixed value of the spatial variable. A specific feature of the obtained frequency responses are the oscillations, closely associated with the presence of the exponential and hyperbolic functions which are periodic along the imaginary axis.

The transfer function representation discussed here has been obtained based on the system of hyperbolic equations in the decoupled canonical form. Thus, the use of the word "general" in the paper title might appear to be somewhat excessive. However, it can be shown that the transfer function matrix for the case of the strongly coupled system can be obtained from its decoupled counterpart via relatively simple transformations, giving as a result transfer functions formulated directly in their physical context. The problem will be addressed in one of the future articles together with some practical examples such as, e.g., electrical transmission line or transport pipeline.

The future works could also include, e.g., consideration of the effects of the boundary feedback and/or reflection on the transfer function description, as well as determination of a general form of the spatio-temporal responses for the individual transfer function channels, both for the congruent and incongruent boundary inputs. Another issue to be thoroughly examined, which is very important from the control synthesis point of view, is selecting an appropriate method for the transfer functions and spatio-temporal responses approximation using finite-dimensional models. The analysis of the approximated models can be difficult since the irrational transfer functions presented here are not nuclear in general, i.e., they do not have a nuclear Hankel operator. It stems from the fact that the semigroup $T(t)$ considered is stable but not analytic, as shown, e.g., by Curtain and Sasane (2001). Therefore, no theoretical upper bound is known for the $H_{\infty}$ errors in the rational approximants produced by truncated balanced realizations and optimal Hankel-norm approximation (Sasane, 2002). In the non-linear case, the approximated models could be based, e.g., on the approximation properties of artificial neural networks (Bartecki, 2010; 2012a).

\section{Acknowledgment}

The author gratefully acknowledges helpful comments from the anonymous reviewers.

\section{References}

Ancona, F. and Coclite, G.M. (2005). On the boundary controllability of first-order hyperbolic systems, Nonlinear Analysis: Theory, Methods \& Applications 63(5-7): e1955-e1966.

Arbaoui, M.A., Vernieres-Hassimi, L., Seguin, D. and Abdelghani-Idrissi, M.A. (2007). Counter-current tubular heat exchanger: Modeling and adaptive predictive functional control, Applied Thermal Engineering 27(13): 2332-2338.

Bartecki, K. (2007). Comparison of frequency responses of parallel- and counter-flow type of heat exchanger, Proceedings of the 13th IEEE IFAC International Conference on Methods and Models in Automation and Robotics, Szczecin, Poland, pp. 411-416.

Bartecki, K. (2009). Frequency- and time-domain analysis of a simple pipeline system, Proceedings of the 14th IEEE IFAC International Conference on Methods and Models in Automation and Robotics, Międzyzdroje, Poland, pp. 366-371.

Bartecki, K. (2010). On some peculiarities of neural network approximation applied to the inverse kinematics problem, Proceedings of the Conference on Control and FaultTolerant Systems, Nice, France, pp. 317-322.

Bartecki, K. (2011). Approximation of a class of distributed parameter systems using proper orthogonal decomposition, Proceedings of the 16th IEEE International Conference on Methods and Models in Automation and Robotics, Międzyzdroje, Poland, pp. 351-356.

Bartecki, K. (2012a). Neural network-based PCA: An application to approximation of a distributed parameter system, in L., Rutkowski, M., Korytkowski, R., Scherer, R., Tadeusiewicz, L. Zadeh and J. Zurada (Eds.), Artificial Intelligence and Soft Computing, Lecture Notes in Computer Science, Vol. 7267, Springer, Berlin/Heidelberg, pp. 3-11.

Bartecki, K. (2012b). PCA-based approximation of a class of distributed parameter systems: Classical vs. neural network approach, Bulletin of the Polish Academy of Sciences: Technical Sciences 60(3): 651-660.

Bartecki, K. and Rojek, R. (2005). Instantaneous linearization of neural network model in adaptive control of heat exchange process, Proceedings of the 11th IEEE International Conference on Methods and Models in Automation and Robotics, Międzyzdroje, Poland, pp. 967-972.

Bounit, H. (2003). The stability of an irrigation canal system, International Journal of Applied Mathematics and Computer Science 13(4): 453-468.

Callier, F.M. and Winkin, J. (1993). Infinite dimensional system transfer functions, in R.F, Curtain, A. Bensoussan and J.L. Lions (Eds.), Analysis and Optimization of Systems: State and Frequency Domain Approaches for Infinite-Dimensional Systems, Lecture Notes in Control and Information Sciences, Vol. 185, Springer, Berlin/Heidelberg, pp. 75-101.

Cheng, A. and Morris, K. (2003). Well-posedness of boundary control systems, SIAM Journal on Control and Optimization 42(4): 1244-1265.

Chentouf, B. and Wang, J.M. (2009). Boundary feedback stabilization and Riesz basis property of a 1-D first order hyperbolic linear system with $L^{\infty}$-coefficients, Journal of Differential Equations 246(3): 1119-1138. 
Christofides, P.D. and Daoutidis, P. (1997). Finite-dimensional control of parabolic PDE systems using approximate inertial manifolds, Journal of Mathematical Analysis and Applications 216(2): 398-420.

Christofides, P.D. and Daoutidis, P. (1998a). Distributed output feedback control of two-time-scale hyperbolic PDE systems, Applied Mathematics and Computer Science 8(4): 713-732.

Christofides, P.D. and Daoutidis, P. (1998b). Robust control of hyperbolic PDE systems, Chemical Engineering Science 53(1): 85-105.

Contou-Carrere, M.N. and Daoutidis, P. (2008). Model reduction and control of multi-scale reaction-convection processes, Chemical Engineering Science 63(15): 4012-4025.

Coron, J., d'Andrea Novel, B. and Bastin, G. (2007). A strict Lyapunov function for boundary control of hyperbolic systems of conservation laws, IEEE Transactions on Automatic Control 52(1): 2-11.

Curtain, R.F. (1988). Equivalence of input-output stability and exponential stability for infinite-dimensional systems, Mathematical Systems Theory 21(1): 19-48.

Curtain, R.F., Logemann, H., Townley, S. and Zwart, H. (1992). Well-posedness, stabilizability, and admissibility for Pritchard-Salamon systems, Journal of Mathematical Systems, Estimation, and Control 4(4): 1-38.

Curtain, R.F. and Sasane, A.J. (2001). Compactness and nuclearity of the Hankel operator and internal stability of infinite-dimensional state linear systems, International Journal of Control 74(12): 1260-1270.

Curtain, R.F. and Weiss, G. (2006). Exponential stabilization of well-posed systems by colocated feedback, SIAM Journal on Control and Optimization 45(1): 273-297.

Curtain, R.F. and Zwart, H. (1995). An Introduction to InfiniteDimensional Linear Systems Theory, Springer-Verlag, New York, NY.

Curtain, R. and Morris, K. (2009). Transfer functions of distributed parameters systems: A tutorial, Automatica 45(5): 1101-1116.

Delnero, C.C., Dreisigmeyer, D., Hittle, D.C., Young, P.M., Anderson, C.W. and Anderson, M.L. (2004). Exact solution to the governing PDE of a hot water-to-air finned tube cross-flow heat exchanger, HVAC\&R Research 10(1): 21-31.

Diagne, A., Bastin, G. and Coron, J.-M. (2012). Lyapunov exponential stability of 1-D linear hyperbolic systems of balance laws, Automatica 48(1): 109-114.

Ding, L., Johansson, A. and Gustafsson, T. (2009). Application of reduced models for robust control and state estimation of a distributed parameter system, Journal of Process Control 19(3): 539-549.

Dooge, J.C.I. and Napiorkowski, J.J. (1987). The effect of the downstream boundary conditions in the linearized St Venant equations, The Quarterly Journal of Mechanics and Applied Mathematics 40(2): 245-256.
Dos Santos, V., Bastin, G., Coron, J.-M. and d'Andréa Novel, B. (2008). Boundary control with integral action for hyperbolic systems of conservation laws: Stability and experiments, Automatica 44(5): 1310-1318.

Evans, L.C. (1998). Partial Differential Equations, American Mathematical Society, Providence, RI.

Filbet, F. and Shu, C.-W. (2005). Approximation of hyperbolic models for chemosensitive movement, SIAM Journal on Scientific Computing 27(3): 850-872.

Friedly, J.C. (1972). Dynamic Behaviour of Processes, Prentice Hall, New York, NY.

Górecki, H., Fuksa, S., Grabowski, P. and Korytowski, A. (1989). Analysis and Synthesis of Time Delay Systems, Wiley, New York, NY.

Grabowski, P. and Callier, F.M. (2001a). Boundary control systems in factor form: Transfer functions and input-output maps, Integral Equations and Operator Theory 41(1): 1-37.

Grabowski, P. and Callier, F.M. (2001b). Circle criterion and boundary control systems in factor form: Input-output approach, International Journal of Applied Mathematics and Computer Science 11(6): 1387-1403.

Gvozdenac, D.D. (1990). Transient response of the parallel flow heat exchanger with finite wall capacitance, Archive of Applied Mechanics 60(7): 481-490.

Jacob, B. and Zwart, H.J. (2012). Linear Port-Hamiltonian Systems on Infinite-dimensional Spaces, Operator Theory: Advances and Applications, Vol. 223, Springer-Verlag, Basel

Jones, B.L. and Kerrigan, E.C. (2010). When is the discretization of a spatially distributed system good enough for control? Automatica 46(9): 1462-1468.

Jovanović, M.R. and Bamieh, B. (2006). Computation of the frequency responses for distributed systems with one spatial variable, Systems Control Letters 55(1): 27-37.

Kowalewski, A. (2009). Time-optimal control of infinite order hyperbolic systems with time delays, International Journal of Applied Mathematics and Computer Science 19(4): 597-608, DOI: 10.2478/v10006-009-0047-x.

Li, H.-X. and Qi, C. (2010). Modeling of distributed parameter systems for applications: A synthesized review from time-space separation, Journal of Process Control 20(8): 891-901.

Litrico, X. and Fromion, V. (2009a). Boundary control of hyperbolic conservation laws using a frequency domain approach, Automatica 45(3): 647-656.

Litrico, X. and Fromion, V. (2009b). Modeling and Control of Hydrosystems, Springer, London.

Litrico, X., Fromion, V. and Scorletti, G. (2007). Robust feedforward boundary control of hyperbolic conservation laws, Networks and Heterogeneous Media 2(4): 717-731.

Lumer, G. and Phillips, R.S. (1961). Dissipative operators in a Banach space, Pacific Journal of Mathematics 11(2): 679-698. 
Maidi, A., Diaf, M. and Corriou, J.-P. (2010). Boundary control of a parallel-flow heat exchanger by input-output linearization, Journal of Process Control 20(10): 1161-1174.

Mattheij, R.M.M., Rienstra, S.W. and ten Thije Boonkkamp, J.H.M. (2005). Partial Differential Equations: Modeling, Analysis, Computation, Society for Industrial and Applied Mathematics, Philadelphia, PA.

Miano, G. and Maffucci, A. (2001). Transmission Lines and Lumped Circuits, Academic Press, San Diego, CA.

Park, H.M. and Cho, D.H. (1996). The use of the Karhunen-Loève decomposition for the modeling of distributed parameter systems, Chemical Engineering Science 51(1): 81-98.

Patan, M. (2012). Distributed scheduling of sensor networks for identification of spatio-temporal processes, International Journal of Applied Mathematics and Computer Science 22(2): 299-311, DOI: 10.2478/v10006-012-0022-9.

Phillips, R.S. (1957). Dissipative hyperbolic systems, Transactions of the American Mathematical Society 86(1): 109-173.

Pritchard, A.J. and Salamon, D. (1984). The linear quadratic optimal control problem for infinite dimensional systems with unbounded input and output operators, in F. Kappel and W. Schappacher (Eds.), Infinite-Dimensional Systems, Lecture Notes in Mathematics, Vol. 1076, Springer, Berlin/Heidelberg, pp. 187-202.

Rabenstein, R. (1999). Transfer function models for multidimensional systems with bounded spatial domains, Mathematical and Computer Modelling of Dynamical Systems 5(3): 259-278.

Rauch, J. and Taylor, M. (1974). Exponential decay of solutions to hyperbolic equations in bounded domains, Indiana University Mathematical Journal 24(1): 79-86.

Salamon, D. (1987). Infinite dimensional linear systems with unbounded control and observation: A functional analytic approach, Transactions of the American Mathematical Society 300(2): 383-431.

Sasane, A. (2002). Hankel Norm Approximation for Infinitedimensional Systems, Springer-Verlag, Berlin.

Staffans, O.J. and Weiss, G. (2000). Transfer functions of regular linear systems, Part II: The system operator and the Lax-Phillips semigroup, Transactions of the American Mathematical Society 354(8): 3229-3262.

Strikwerda, J. (2004). Finite Difference Schemes and Partial Differential Equations, Society for Industrial and Applied Mathematics, Philadelphia, PA.

Sutherland, J.C. and Kennedy, C.A. (2003). Improved boundary conditions for viscous, reacting, compressible flows, Journal of Computational Physics 191(2): 502-524.
Tucsnak, M. and Weiss, G. (2006). Passive and Conservative Linear Systems, Nancy University, Nancy.

Tucsnak, M. and Weiss, G. (2009). Observation and Control for Operator Semigroups, Birkhäuser, Basel.

Uciński, D. (2012). Sensor network scheduling for identification of spatially distributed processes, International Journal of Applied Mathematics and Computer Science 22(1): 25-40, DOI: $10.2478 / \mathrm{v} 10006-012-0002-0$.

Weiss, G. (1994). Transfer functions of regular linear systems, Part I: Characterizations of regularity, Transactions of the American Mathematical Society 342(2): 827-854.

$\mathrm{Wu}$, W. and Liou, C.-T. (2001). Output regulation of two-time-scale hyperbolic PDE systems, Journal of Process Control 11(6): 637-647.

Xu, C.-Z. and Sallet, G. (2002). Exponential stability and transfer functions of processes governed by symmetric hyperbolic systems, ESAIM: Control, Optimisation and Calculus of Variations 7: 421-442.

Zavala-Río, A., Astorga-Zaragoza, C.M. and Hernández-González, O. (2009). Bounded positive control for double-pipe heat exchangers, Control Engineering Practice 17(1): 136-145.

Zwart, H. (2004). Transfer functions for infinite-dimensional systems, Systems and Control Letters 52(3-4): 247-255.

Zwart, H., Gorrec, Y.L., Maschke, B. and Villegas, J. (2010). Well-posedness and regularity of hyperbolic boundary control systems on a one-dimensional spatial domain, ESAIM: Control, Optimisation and Calculus of Variations 16(04): 1077-1093.

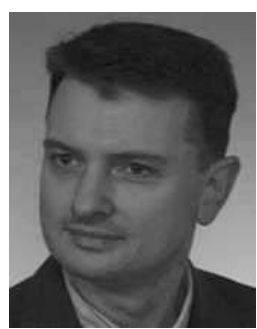

Krzysztof Bartecki received his M.Sc. and $\mathrm{Ph} . \mathrm{D}$. degrees in electrical engineering from the Faculty of Electrical Engineering, Automatic Control and Computer Science, Opole University of Technology, in 1996 and 2004, respectively. $\mathrm{He}$ is an assistant professor at the Institute of Control and Computer Engineering. He has authored or co-authored some 40 papers, concerning mainly the modeling of distributed parameter systems as well as application of artificial neural networks in identification and control of dynamic objects.

Received: 25 April 2012

Revised: 9 January 2013 